\title{
Optimized square Fresnel zone plates for microoptics applications
}

\author{
José María Rico-García ${ }^{1}$, Francisco Javier Salgado-Remacha ${ }^{1}$, Luis Miguel Sanchez-Brea ${ }^{1}$ and \\ Javier Alda ${ }^{2}$ \\ Applied Optics Complutense Group, \\ Optics Department, University Complutense of Madrid, \\ ${ }^{1}$ Physics Faculty, Av Complutense, s/n, 28040 \\ ${ }^{2}$ School of Optics. Av. Arcos del Jalón s/n, 28037 Madrid, Spain \\ Phone: +34 9139468 72, Fax: +34 913946885
}

E-mail: jmrico@fis.ucm.es, fjsalgado@fis.ucm.es, sanchezbrea@fis.ucm.es, j.alda@opt.ucm.es

\begin{abstract}
Polygonal Fresnel zone plates with a low number of sides have deserved attention in micro and nanoptics, because they can be straightforwardly integrated in photonic devices, and, at the same time, they represent a balance between the high-focusing performance of a circular zone plate and the easiness of fabrication at micro and nano-scales of polygons. Among them, the most representative family are Square Fresnel Zone Plates (SFZP). In this work, we propose two different customized designs of SFZP for optical wavelengths. Both designs are based on the optimization of a SFZP to perform as close as possible as a usual Fresnel Zone Plate. In the first case, the criterion followed to compute it is the minimization of the difference between the area covered by the angular sector of the zone of the corresponding circular plate and the one covered by the polygon traced on the former. Such a requirement leads to a customized polygon-like Fresnel zone. The simplest one is a square zone with a pattern of phases repeating each five zones. On the other hand, an alternative SFZP can be designed guided by the same criterion but with a new restriction. In this case, the distance between the borders of different zones remains unaltered. A comparison between the two lenses is carried out. The irradiance at focus is computed for both and suitable merit figures are defined to account for the difference between them
\end{abstract}

\section{INTRODUCTION}

One of the most prominent devices in the field of diffractive optics is the Fresnel Zone Plate (FZP). Different designs have been proposed along the years to account for a plethora of application both in micro and nanoptics [1,2]. The FZP usually works as a lens on 2D geometries, yet extensions to 3D problems have been proposed [3]. The properties of a usual FZP lie on the aperture splitting into a semiperiodic set of zones [3]. If the phase difference between the wavelets stemming from contiguous zones is compensated properly at the observation plane by a customized phase or amplitude mask, the FZP acts as focusing element. Even more, further sub-zoning of the aperture is possible, thus improving the performance and versatility of the original FZP. The subsequent compensating mask is more complex as the number of different phase levels increases. Nonetheless, not only there have been proposals regarding variations over the phase distribution of the zones on the FZP, but also proposals regarding changes in their geometry. Perhaps, one of the most appealing approaches for micro and nanoptics are Square Fresnel Zone Plates (SFZP) [4,5]. Although their focusing performance is not as good as a FZP, the easiness to fabricate them at micro and nanoscales make them a serious alternative to common FZPs for their integration in optoelectronic and integrated optical circuits. Furthermore, due to their geometry, they are more robust to fabrications imperfections than a FZP at these scales.

In this paper, we describe the theoretical basis for a customized design of a SFZP at visible wavelengths in section 2. We will focus on two slightly different families of SFZPs, i.e, those showing a periodic pattern of phases as the number of zones increases and those characterized by zones with equal width. An analytical approach to compute the

Modeling Aspects in Optical Metrology II, edited by Harald Bosse, Bernd Bodermann, Richard M. Silver, Proc. of SPIE Vol. 7390, 739011 - @ 2009 SPIE · CCC code: 0277-786X/09/\$18 · doi: 10.1117/12.827546 
corresponding phases for a given zone will be depicted in both cases. Section 3 is devoted to the numerical computation of the focusing features for both kinds of lenses. A numerical algorithm based on the RayleighSommerfeld formula to propagate the field until the observation plane is presented [6]. The outputs from this algorithm will be compared with those of Section 4, where the experimental set-up whose main tool is a reflective Spatial Light Modulator (SLM) will be explained. Also, a comparison between the main features on both kinds of lenses will be carried out, so as to asses their performance as focusing diffractive elements. Finally, all the results will be summarized in Section 5 .

\section{2.- THEORETICAL BASIS FOR SQUARE FRESNEL ZONE DESIGN}

The goal pursued in fixing the radii of the standard circular Fresnel Zone Plate is to achieve contiguous circular zones with a phase difference of $\pi$. This difference is compensated at the focus plane by a suitable mask. In order to get such a difference for a number of zones $m$, a work wavelength $\lambda$ and a focus length $f^{\prime}$, the relation between these parameters and the radii of the Fresnel zones should be [3]

$$
r_{m}=\sqrt{f^{\prime} \lambda m+\left(\frac{\lambda m}{2}\right)^{2}}
$$

One of the main features of a FZP is the quadratic dependence of the irradiance at focus with $\mathrm{m}$. As it will be seen below, this is not the case for a SFZP, where the dependence is not quadratic. In fact, this trend for a high number of zones is different for the two families of SFZPs we are analyzing here.

Regarding the first family of SFZPs, a fundamental requirement to achieve a performance similar to that of a FZP is that the areas of the corresponding zones must be as equal as possible. This, of course, cannot be fully achieved due to the mismatch between the geometries of both kinds of lenses. Nonetheless, the area difference between the circular zone and the superimposed polygonal zone can be minimized in terms of the apothem $x_{m}$ of the polygon [4]. Such a constriction leads to a relation between the apothem, the number of sides of the polygon, $\mathrm{s}$, and the radius of the FZP $r_{m}$ for a polygon-like Fresnel Lens

$$
x_{m}=\frac{r_{m}}{\sqrt{1+\frac{\tan ^{2}(\pi / s)}{4}}}
$$

For a square, the relation between the apothem and the radii of the SFZP is

$$
x_{m}=\frac{2 r_{m}}{\sqrt{5}}
$$

since $s=4$. The phase at focus for the m-zone that must be compensated can be computed from the knowledge of the amplitude at focus due to the secondary wavelets coming from this zone. This quantity is equal to

$$
A_{m}=\int_{-x_{m+1}}^{x_{m+1}} \int_{x_{m+1}}^{x_{m+1}} E(x, y) d x d y-\int_{-x_{m}}^{x_{m}} \int_{-x_{m}}^{x_{m}} E(x, y) d x d y
$$

where $E(x, y)$ is the field amplitude at point $(x, y)$ in the focus plane, and the equation above accounts for the contribution of the m-zone to the field at focus. The field at the focal point is computed throughout using the previous integral with the following dependence of the electric field,

$$
E(x, y)=\frac{1}{r(x, y)}\left[\frac{1}{2}\left(1+\frac{f^{\prime}}{r(x, y)}\right)\right] \exp \left[-i \frac{2 \pi n}{\lambda_{o}} r(x, y)\right]
$$

where $r(x, y)$ is the distance between the points $(x, y)$ and the focal plane. $f^{\prime}$ is the focus and $\mathrm{n}$ is the refractive index of the medium after the SFZP.

If the phase difference between contiguous zones is computed for the SFZP, it can be seen that is constant and equal to $\phi=4 \pi / 5$, giving rise, but for the central zone, to a periodic pattern of phases that are continuously reproduced as 
the number of zones increases. Both the size and the phase for the central zone are calculated accordingly to the nominal focal length of the SFZP.

The most remarkable aspect of this family of diffractive lenses is the linear growth of the power with the number of zones, at least for those lenses with a low number of zones.

The second family of SFZP is obtained from a more rudimentary calculation. The main problem obtained from the first family is that, when realizing experimentally the previous mask using a Spatial Light Modulator (SLM), most of the borders between contiguous zones are located in the middle of one pixel of the SLM. Then, some corrections of the phase should be done to practically compensate this mixed contribution. A simple solution for this problem is to consider the width of the square zone as constant, and even better, as an integer multiple of the pixel size. In such a case, each square zone contains and integer number of pixels, avoiding phase compensation between adjacent zones.

The calculation of the phase for each zone is quite simple. The integral used previously is again evaluated for each zone. The phase obtained for the contribution of every zone is properly compensated at the SFZP plane as a phase mask. This phase mask is realized by using a SLM.

\section{3.- RAYLEIGH-SOMMERFELD ANALYSIS OF THE LENS PERFORMANCE}

A more in-deep analysis of the lens performance involves the use of the Rayleigh-Sommerfeld propagation integral for the masks obtained from the algorithms mentioned above,

$$
U(x, y ; z)=\int U(\varsigma, \eta, 0) \frac{\exp (i k r)}{2 \pi r} \frac{z}{r}\left(\frac{1}{r}-i k\right) d \varsigma d \eta
$$

where $U(x, y ; z)$ stands for the amplitude of the field at plane $\mathrm{z} ;(\varsigma, \eta)$ are the coordinates at the mask plane and $\mathrm{k}$ is the wavenumber. The integral is computed on the mask aperture. We have computed the field aided by an FFT version of the previous integral [6]. To get a reliable calculation of the field at focus, a re-sampling procedure on the computed quantities has been carried out. The results from this method have been compared with those coming from the measurements in the next section.

\section{4.- EXPERIMENTAL SET-UP AND RESULTS}

A He-Ne laser $(\lambda=632.8 \mathrm{~nm}$ ) generates a beam impinging on a Spatial Light Modulator (SLM) that will be used to simulate the transmittance of the SFZPs. The SLM is a Holoeye LCR-2500 reflective modulator, with $1024 \times 768$ pixels ( 8 bits resolution). It is driven by the graphic board of a PC. The SLM plays the role of a secondary monitor. in charge of creating the desired distribution at focus of the lens under analysis.

. Although the SLM works fine, due to the fabrication process of its pixels, it adds a considerable amount of aberrations to the wavefront of the reflected beam. This deviation from the ideal performance is model-dependent. Hence, to circumvent this problem, we have taken advantage of a successful technique employed in this kind of device. $[7,8,9]$. In our case, the SLM contributes to the phase added by the SZP with a significant amount of coma. Therefore, the phase of a suitable astigmatic lens is added to the phase map of the Fresnel lens to eliminate the astigmatism. The phase of the correction lens is

$$
\phi_{\text {astigmatic }}=\frac{\pi}{\lambda}\left[\left(\frac{x \cos \theta+y \sin \theta}{\sqrt{f_{1}}}\right)^{2}+\left(\frac{-x \sin \theta+y \cos \theta}{\sqrt{f_{2}}}\right)^{2}\right],
$$

where the parameters $f_{1}$ and $f_{2}$ are the Sturm foci and $\theta$ is the angle between the lens axes and the laboratory axes. These parameters are experimentally adjusted until no aberrations are observed: $f_{1}=14 \mathrm{~m}, f_{1}=8 \mathrm{~m}$ and $\theta=-167.7^{\circ}$. Two examples of phase maps for the two families of SFZPs investigated are plotted in Figure 1 for a different number of zones. As it can be noticed out from Figure 1, the relation between the area covered by the lens and the number of zones is different for each lens. 

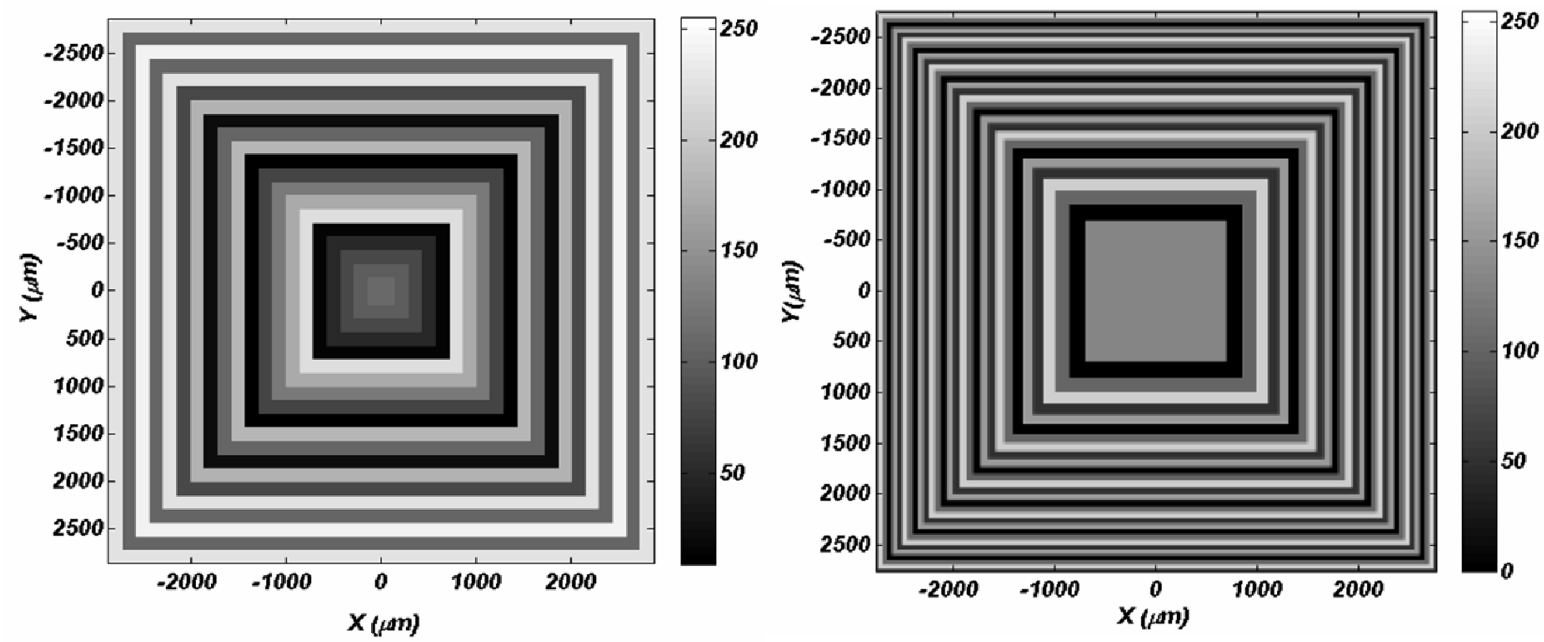

Figure 1: On the left, 20 zone equal width SFZP. On the right, 30 zone SFZP with customized periodic pattern of phases according to the computation method mentioned in Section 1

The experimental Set-Up is shown in Figure 2. Two lineal polarizers, control the beam intensity and the polarization state of the incoming beam. Then a spatial filter is employed to make sure that the beam impinging on the SML is Gaussian. A collimating lens is placed to properly illuminate the whole plane of the SML. We use a 50/50 beamsplitter, located at $45^{\circ}$. Therefore the SLM is operated in the standard normal incidence configuration. The reflected light modulated by the device is redirected by means of the beam splitter to a camera, with a pixel pitch of $6 \mu \mathrm{m}$. The camera travels along the optical axis. Thus, any relevant quantity in the propagation of the focused beam can be measured at any work plane after the beam splitter.

In order to adapt the the square Fresnel zones geometry, as much as possible to the SLM, we have designed them taking into consideration the pixel size and period, which is $19 \mu \mathrm{m}$ along a rectangular grid. Consequently, the pixels are oriented in the regular grid with this period for the directions $x$ and $y$.

An extensive analysis of the performance of the two families of SFZPs has been done. We have measured the power at focus for a set of lenses of the same family with a number of zones varying from one lens to another. At the same time, the Rayleigh-Sommerfeld integral approach has been employed to get the corresponding theoretical curve. Moreover, a linear fit for both families have been achieved when the number of zones is low. The results, along with the predictions from the simulation are shown in Figure 3 for SFZP with equal area between zones and for SFZP with a periodic pattern of phase

The set of SFZP for both families are characterized by a focal distance $f^{\prime}=0.5$ for SFZP with a periodic pattern of phases and $f^{\prime}=0.3$ for SFZP with equal width between zones. The irradiance has been normalized to its maximum in both cases.

In the first case, if we surmise a dependence $I=I_{0}+\alpha m$, the parameters of the fit to the curve are $I_{0}^{\text {periodic }}=0.138 \pm 0.005$ and $\alpha^{\text {periodic }}=0.0159 \pm 0.0003$. The linear regression coefficient is $R^{\text {periodic }}=0.9612$.

In the second, the corresponding values are $I_{0}^{\text {equal }}=-0.10 \pm 0.01$, and $\alpha^{\text {equal }}=0.0312 \pm 0.0003$. The linear regression coefficient is $R^{\text {equal }}=0.9417$. These results demonstrate that the linear trend is clearer in the first family of SFZP than in the second. 


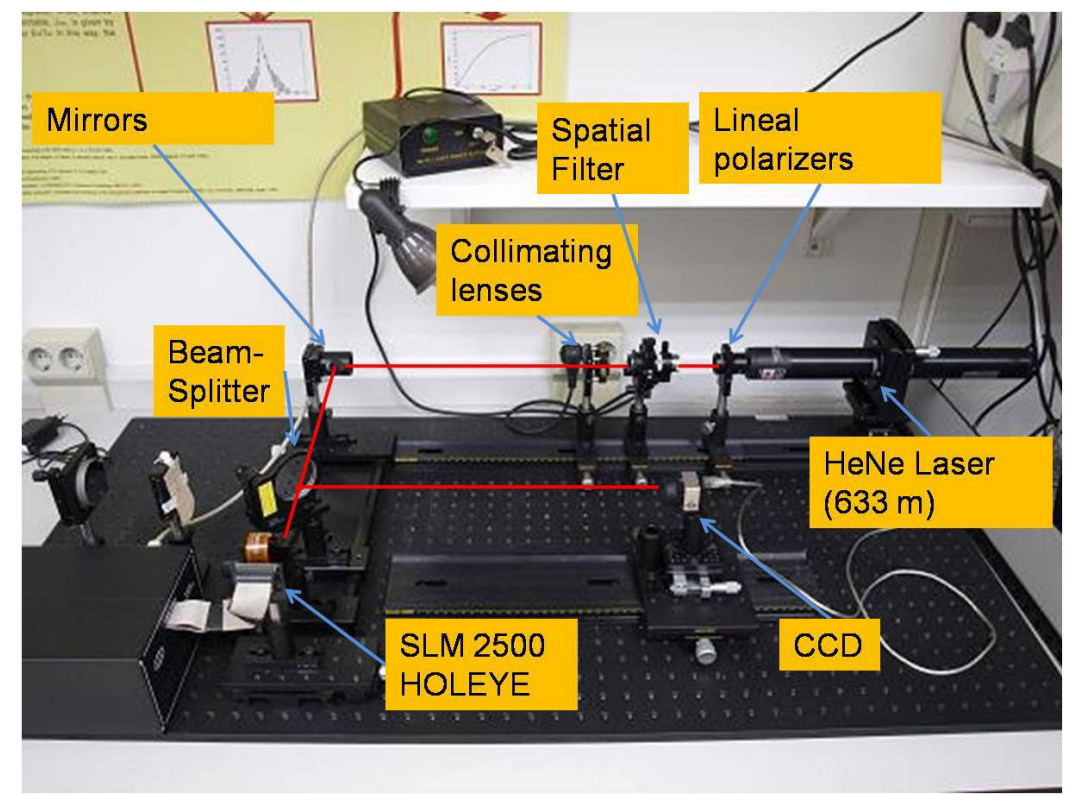

Figure 2: Picture of the experimental set-up. All the relevant elements have been remarked.

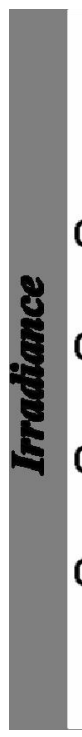

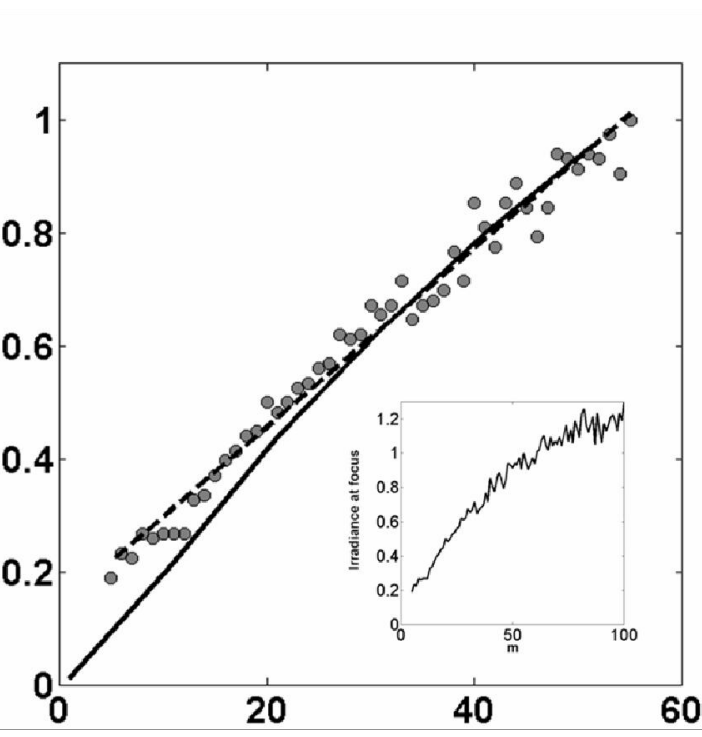

Number of zones

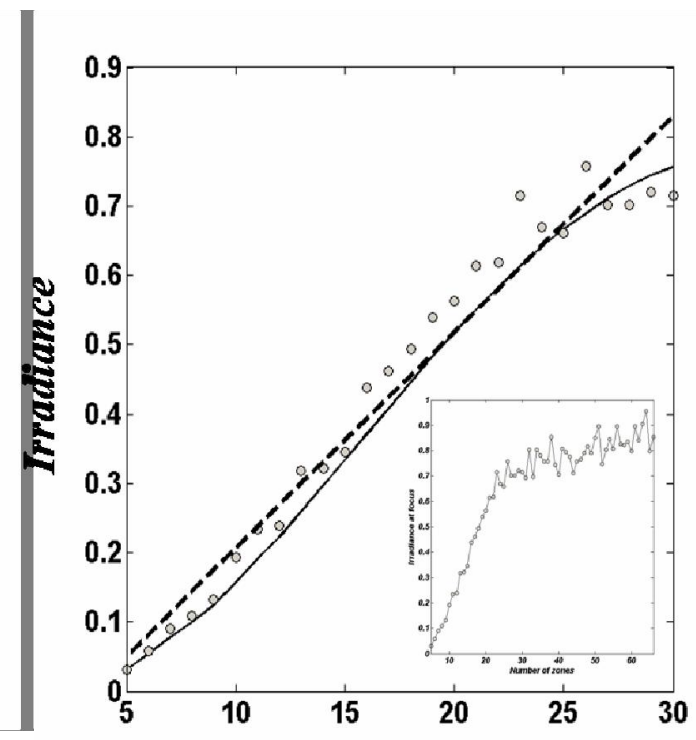

Number of zones

Figure 3: Measurement (dots), computation (continuous line) and linear fit (dashed line) to the dependence of the irradiance at focus vs number of zones for the two of SFZP. On the left, SFZP with a periodic pattern of phases. On the right, SFZP with equal-width zones. The irradiance has been normalized to its maximum in both cases

To further enlighten the behaviour of these optical devices, different images of the intensity at focus for each kind of lens has been recorded in the CCD camera. In Figure 4 and Figure 5 is displayed the Rayleigh-Sommerfeld predictions for the two families. Figures 6 and 7 depict the experimental results for the phase distributions projected on the SML for Figures 4 and 5. 


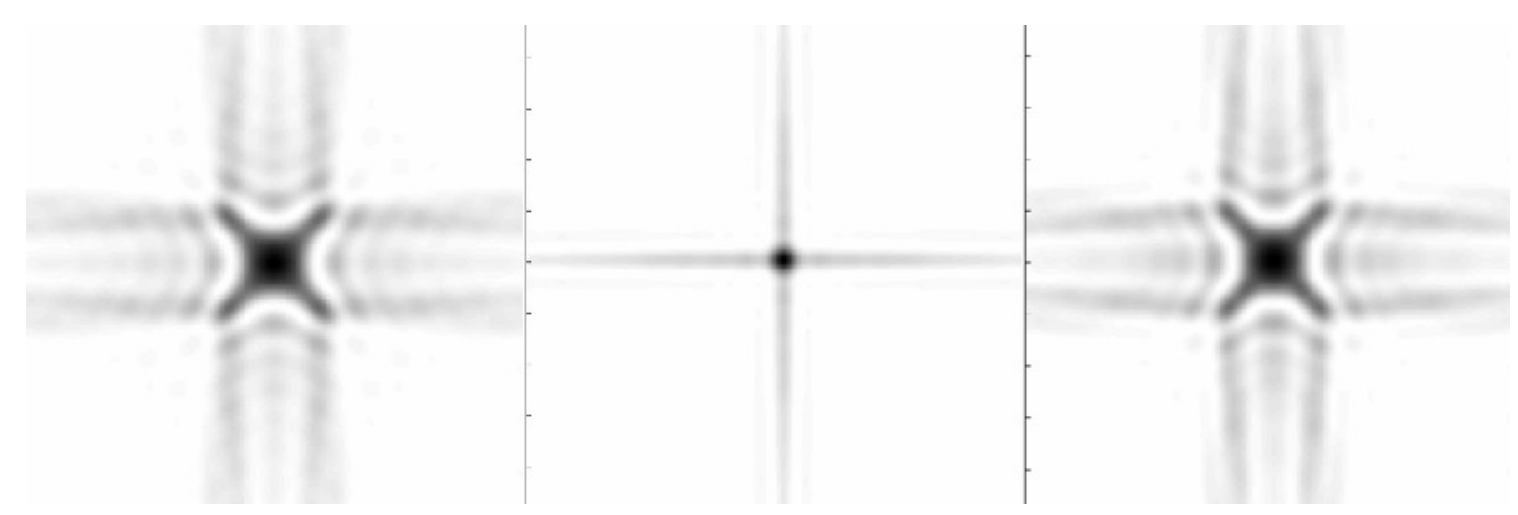

Figure 4: Computed equal-width SFZP intensity patterns for a typical lens of the family. ( $\mathrm{f}^{\prime}=0.5$, Number of Zones: 30 , for $\lambda=633 \mathrm{~nm}$ ). From left to right: Prefocal, focal and post-focal planes of observation

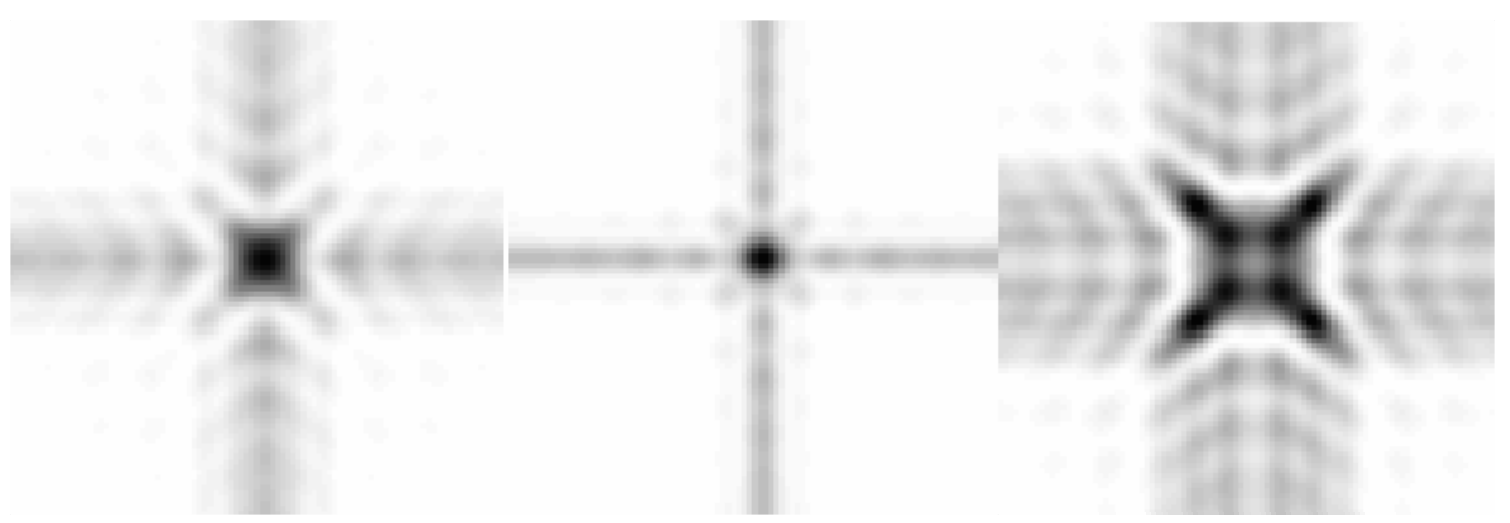

Figure 5: Computed customized SFZP intensity patterns for a typical lens of the family. ( $\mathrm{f}^{\prime}=0.5$, Number of Zones: 100 , for $\lambda=633 \mathrm{~nm}$ ). From left to right: Prefocal, focal and post-focal planes of observation

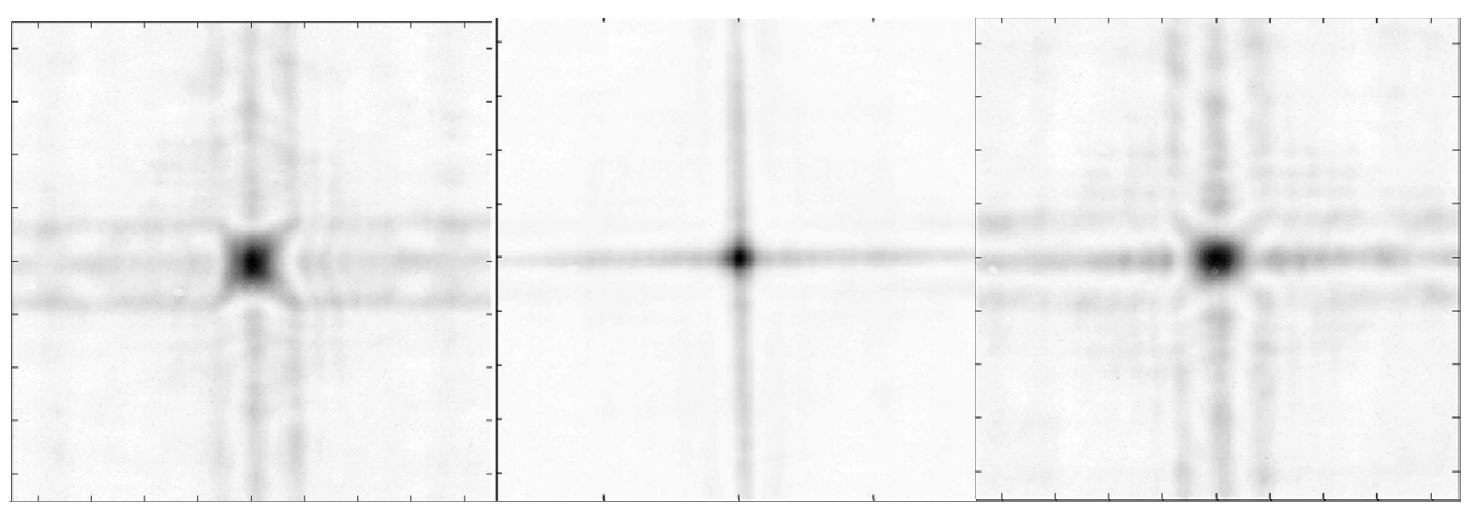

Figure 6: Measured equal-width SFZP intensity patterns for a typical lens of the family. ( $\mathrm{f}^{\prime}=0.5$, Number of Zones: 60 , for $\lambda=633 \mathrm{~nm}$ ). From left to right: Prefocal, focal and post-focal planes of observation 


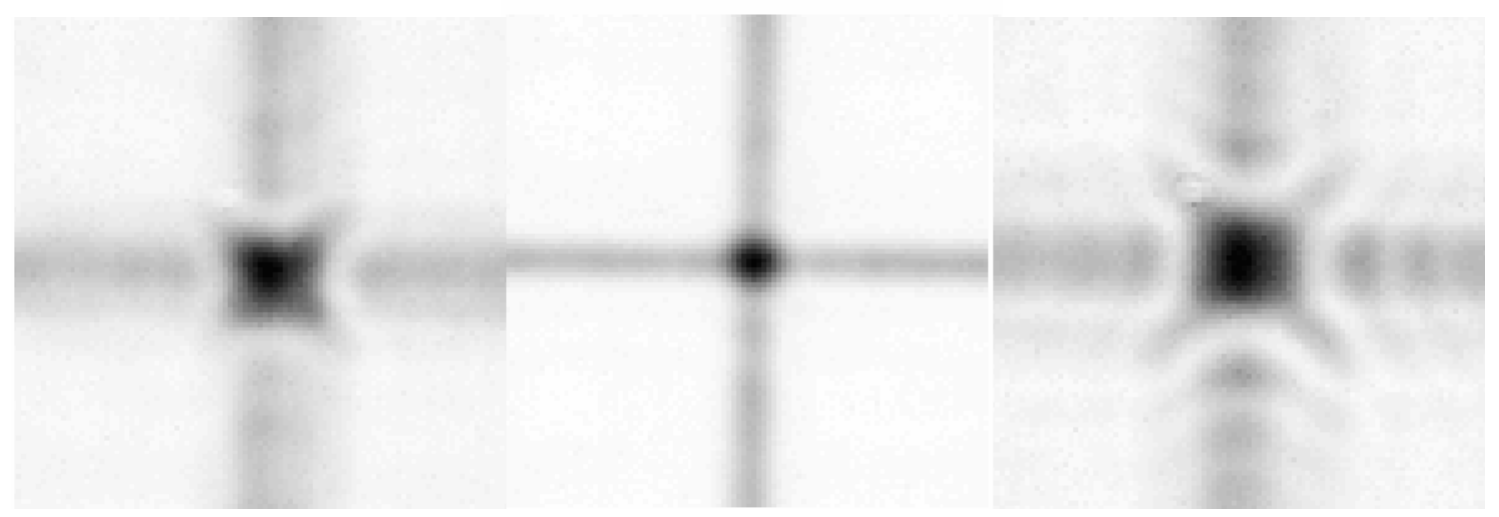

Figure 7: Measured customized SFZP intensity patterns for a typical lens of the family. ( $\mathrm{f}^{\prime}=0.5$, Number of Zones: 100 , for $\lambda=633 \mathrm{~nm}$ ). From left to right: Prefocal, focal and post-focal planes of observation

As it can be noticed out from the collected data, theory and experiments fit well for both classes of lenses. It is worthwhile to mention the different trend in the power at focus for the two families of lenses as the numbers of zones grow up.

Another point to be remarked is the symmetry of the intensity spatial distribution for different SFZPs. Despite the fact there is no symmetry between the post-focal and the pre-focal plane for the customized SFZP family, this not the case for an equal-width SFZP.

However, but for the curve of intensity at focus vs number of zones, both kinds of lenses perform very similar.

\section{CONCLUSIONS}

In this communication, we have compared two different realizations of Fresnel Zone Plates characterized by squareshaped zones. These are two distinct families, those who have been computed minimizing the area between each zone and its associated circular sector in a standard FZP, and those who are designed with equal-width zones. They show different behaviour as the number of zones increases. However, for a low number of zones, both depict a linear performance. Finally, the agreement between the measurements made with the help of an SML and the computations get from the Rayleigh-Sommerfeld integral proves the feasibility of these Diffractive Optical Elements for Micro and Nanooptics applications.

\section{ACKNOWLEGMENTS}

This work has been partially supported by the projects TEC2006-01882 of the Ministerio de Educación y Ciencia and Project CCG08-UCM/DPI-3952 of Dirección General de Universidades e Investigación de la Consejería de Educación de la Comunidad de Madrid y Universidad Complutense de Madrid. Salgado-Remacha acknowledges the economical support of "Ministerio de Educación y Ciencia" and the European Social Fund (DPI2005-02860.)

\section{REFERENCES}

[1] F. J. González. J. Alda, B. Illic, G. Boreman, Appl. Opt. 43 (2004) 6067-6073.

[2] J. Ginn, B. Lail, J. Alda, G. Boreman, Opt. Lett. 33 (2008) 779-781

[3] H. D. Hristov, "Fresnel zones in wireless links, zone plate lenses and antennas", Artech House, Norwood, MA, USA, 2000.

[4] J. Alda, G. Boreman, Microwave Opt. Technol. Lett. 50 (2008) 536-541.

[5] I. V. Minin, O. V. Minin, A. Petosa, S. Thirakuone, Microwave Opt. Technol. Lett. 49 (2007) 276-278.

[6] F. Shen, and A. Wang, Appl. Opt. 45 (2006) 1102-1110. 
[7] E. Pleguezuelos, J. Andilla, A. Carnicer, E. Martín-Badosa, S. Vallmitjana, and M. Montes-Usategui, Proc of SPIE 6326 (2006) 63262Q.

[8] J. Otón, P. Ambs, M. S. Millán, and E. Pérez-Cabré., Appl. Opt. 46 (2007) 5667-5679.

[9] M.L. Cordero, D.R. Burnham, C.N. Baroud, D. McGloin, Appl. Phys. Lett. 93 (2008) 034107. 